\title{
3 Research Square

\section{Evaluation of level of expression of microRNA-21 and let-7g in serum and stool of patients with colorectal cancer}

\section{Dara Rahmanpour}

Tabriz University of Medical Sciences

Roya Dolatkhah

Tabriz University of Medical Sciences

Soheil Navidizadeh

Tabriz University of Medical Sciences

Peyman Virani

Tabriz University of Medical Sciences

Nastran Hasanzadeh

Tabriz University of Medical Sciences

Shahryar Hashemzadeh ( $\nabla$ shahryar.hashemzadeh2020@gmail.com )

Tabriz University of Medical Sciences

\section{Research Article}

Keywords: CRC, Real-Time PCR, miR-21, let-7g

Posted Date: November 11th, 2021

DOI: https://doi.org/10.21203/rs.3.rs-1061712/v1

License: () (1) This work is licensed under a Creative Commons Attribution 4.0 International License. Read Full License 


\section{Abstract \\ Background}

MicroRNAs (miRs) are involved in the pathogenesis of various malignancies such as colorectal cancer through regulating multiple cellular processes, including cell proliferation, cell cycle, apoptosis and migrationMiR-21 and let-7 are two important genes that have confirmed in this pathway. The role of the let-7 gene as a gene tumor process in various cancers and the role of miR-21 in the development and progression of cancer has been conclusively identified also this gene has an oncogenic role in various cancers. In this study, the expression patterns of miR-21 and let-7 in serum and stool samples of colorectal cancer patients were evaluated.

\section{Materials and Methods}

During the present study, 120 samples including 40 serum samples of CRC and 40 stool samples from the same patients and 40 healthy samples were collected. After total RNA extraction, real-time PCR was used to measure changes in genes expression. Statistical analysis of data was performed with GraphPad Prism statistical software (Version 6.0) with a significance level of $5 \%$.

\section{Results}

The relative expression level of miR-21 in the serum samples of CRC increased compared to the healthy group, which was statistically significant. On the other hand, the relative expression level of let-7g in the serum samples of CRC showed a significant decrease compared to the healthy sample. In stool samples, the expression changes of either of the two genes were not significant.

\section{Conclusion}

Our findings indicate that the relative expression of miR-21 and let-7g genes can be used as a diagnostic or predictive biomarker in colorectal cancer serum samples. While, this is not the case in stool samples. Moreover, further investigations at the protein level should be performed.

\section{Introduction}

Colorectal cancer is considered one of the most prevalent and lethal malignancies, worldwide [1, 2], responsible for half million deaths annually $[3,4]$. Regardless of recent progresses in cancer diagnosis and treatment, the overall prognosis of colorectal cancer is still poor [5]. A number of these molecular events that occur during this process have been identified, but thousands of other molecules involved in the process remain unknown, and their identification is essential to detect this process as quickly as possible and to develop therapeutic strategies [5]. 
Recent research has shown that abnormal expression and activity of miRNAs are the important events in the initiation and progression (from onset to metastasis) of various cancers, including colorectal cancer. Different miRNAs have been reported to act as oncogenes or suppressor tumors in different cancers [6$11]$.

Let-7 microRNAs are non-encoded RNAs with 22 nucleotides [12]. Let-7 microRNAs are down-regulated in several types of cancers such as CRC, Which affects various stages such as post-transcriptional regulation of target microRNAs, tumorigenesis and progression of colon cancer [13].

Let7g is one of the genes of the Let7 family. In fact, this family of microRNAs is the first family of microRNA genes known in eukaryotes. The role of this family in a variety of cancers has been proven, and these micro RNAs are now recognized not only as tumor suppressors of genes that can serve as biomarkers for cancer, but also as therapeutic targets in a variety of cancers [14-16].

MiR-21 is another miRNA which functions as an oncogenic miRNA and participates in theregulation of numerous cancer-related target genes [17-19]. MiR-21 has been shown to be upregulated through colorectal tumorigenesis, possessing great value as an independent prognostic and predictive biomarker [20-23]. Despite thatan enormous number of investigations have been carried out to inspect the blood levels of circulating miR-21 in cancer patients compared to healthy individuals, the results of these studies on the diagnostic and prognostic potential of circulating miR-21 have been inconsistent [24-26].

A growing body of evidence shows that miRNAs exhibit abnormal expression patterns in peripheral blood samples of patients suffering from CRC, which, in turn, indicates potenatil of these miRNAs to serve as biomarkers predicting CRC incidence and progression [27]. Recent studies in CRC have shown that mir-21 is not only associated with malignancy but also that changes in its expression are associated with drug resistance of colorectal cancer cells [27].

Recently, due to the nature of colorectal cancer, new studies have examined changes in biological molecules, including micro RNAs in the serum and stool of patients with this disease, to find new biomarkers associated with this cancer. Several recent studies have shown changes in the serum levels of miR-21 and let7g in the patients with various cancers, including colorectal cancer, while fewer similar studies have been performed in the stool samples of these patients $[9,28]$. Also, no similar study has been performed in the serum or stool of patients in the study population.

\section{Materials And Methods}

\section{Sample collection}

In the present study with a case-control design, blood and stool samples were collected from 50 patients suffering from CRC before surgery by the surgeon in Imam Reza Hospital, Tabriz University of Medical Sciences. The samples were taken from patients who refer to the hospital from March 2018 to July 2018. The participants received no therapies, including chemotherapy or radiotherapy. Clinicopathological 
information related to each of the available files was reviewed and summarized in Table 1. First, sera were separated from patients' blood samples by centrifugation at $12,000 \mathrm{~g}$ for $10 \mathrm{~min}$ at $4 \circ \mathrm{C}$ and stool samples homogenized to extract RNA. Till RNA extraction process, all samples were instantly transported into Qiagen RNase inhibitor solution (Cat NO: 76104) and stored at $-80^{\circ}$.

Table 1

General Characteristic of Study patients

\begin{tabular}{|c|c|c|c|c|c|}
\hline Parameters & $\begin{array}{l}\text { Number of } \\
\text { Patients }\end{array}$ & $\begin{array}{l}\text { Relative Mir-21 } \\
\text { expression }\end{array}$ & $\begin{array}{l}P \\
\text { value }\end{array}$ & $\begin{array}{l}\text { Relative let-7 } \\
\text { expression }\end{array}$ & $\begin{array}{l}p \\
\text { value }\end{array}$ \\
\hline Age $<50$ years & 12 & $0.64 \pm 0.36$ & & $1.25 \pm 027$ & \\
\hline Age $>50$ years & 28 & $0.95 \pm 0.45$ & & $0.45 \pm 0.16$ & \\
\hline Tumor size $<2 \mathrm{~cm}$ & 26 & $1.14 \pm 0.31$ & & $0.96 \pm 0.41$ & \\
\hline Tumor size $>2 \mathrm{~cm}$ & 14 & $0.46 \pm 0.24$ & & $0.33 \pm 0.09$ & \\
\hline Stage I & 20 & $1.01 \pm 0.42$ & & $0.78 \pm 0.31$ & \\
\hline Stage II and III & 20 & $0.64 \pm 0.35$ & & $0.72 \pm 0.52$ & \\
\hline Grade I & 21 & $0.97 \pm 0.41$ & & $1.01 \pm 0.41$ & \\
\hline Grade II and III & 19 & $0.75 \pm 0.49$ & & $0.44 \pm 0.24$ & \\
\hline $\begin{array}{l}\text { Lymph node } \\
\text { metastasis POS }\end{array}$ & 24 & $0.51 \pm 0.20$ & & $0.47 \pm 0.19$ & \\
\hline $\begin{array}{l}\text { Lymph node } \\
\text { metastasis PO }\end{array}$ & 16 & $0.71 \pm 0.43$ & & $0.91 \pm 0.41$ & \\
\hline
\end{tabular}

\section{Extraction of total RNA}

To isolate total RNA from prepared samples, including serum and stool samples, TRIzol RNA extraction reagent (Cat NO: 11667165001) was used regarding to instructions provided by manufacture. After extraction, The quantity and quality of RNA was evaluated using Nanodrop spectrophotometer (Thermo Fisher Scientific, USA) considering the optical density at $260 \mathrm{~nm}$ and 280 wavelengths. Before complementary DNA synthesis (cDNA) synthesis, extracted RNA samples were stored at $-80^{\circ} \mathrm{C}$ until the.

\section{cDNA synthesis \& real-time PCR:}

To determine miR-21 and let-7g levels in obtained samples, one microgram of extracted RNA was reverse transcribed to CDNA using Exiqon miRCURY ${ }^{\mathrm{TM}}$ LNA $^{\mathrm{TM}}$ miRNA RT Kit regarding to instructions supplied. The T100 Thermocycler system (Bio-Rad, CA, USA) was used to carried out reactions at a final volume of 20 $\mu l$. Then, the evaluation of the mature miRNA levels was performed using the ExiLENT SYBR Green master mix (Exiqon) at a light cycler 96 system (Roche, Germany). The specific primer sets for each miRNA are provided in Table 2. Each reaction was repeated twice (duplicated) and target miRNA 
expression was normalized to $\mathrm{U} 6$ internal control expression levels. The $2^{-\Delta \mathrm{Ct}}$ method $(\Delta \mathrm{Ct}=\mathrm{Ct}$ target gene $-\mathrm{Ct}_{\mathrm{U}}$ ) was used to quantify the relative expression of target miRNAs.

Table 2

primer sequences used for qRT-PCR

\begin{tabular}{|lll|}
\hline miRNA & & Product number \\
\hline Let-7f & Purchased from Exiqon & 202105 \\
\hline U6 & Purchased from Exiqon & 218300 \\
\hline Mir-21 & Purchased from Exiqon & 202,007 \\
\hline
\end{tabular}

\section{Statistical Analyses:}

GraphPad Prism 6 (GraphPad Software, USA) was employed to perform statistical analysis between experimental groups. All data are presented as mean \pm standard deviation. For data with normal distribution and data with non-normal distribution, student's t-test and Mann-Whitney test were used, respectively. It was considered statistically significant if $p$ value was less than 0.05 .

\section{Results}

\section{Mir-21 significant up-regulation in CRC serum samples}

To investigate miR-21 expression levels in a set of internal samples, including $40 \mathrm{CRC}$ and healthy serum and blood samples, qRT-PCR was performed. As illustrated in Fig. 1, the obtained results evidenced that miR-21 was significantly ( $p$ value $=0.0017)$ up-regulated in serum samples of colorectal cancer compared to healthy samples. But in the stool sample of patients with colorectal cancer, no significant change was observed in this gene compared to the healthy group (Pvalue=0.731) (Fig. 2).

\section{Low expression levels of Let-7g in CRC serum samples}

Besides the miR-21 upregulation, qRT-PCR results showed that let-7g expression levels were downregulated in serum samples of CRC patients in comparison to healthy samples ( $p$ value $=0.019)$ that shown in Fig. 3. But in the stool sample of patients with colorectal cancer, no significant change was observed in this gene compared to the healthy group ( $p$ value=0.51) (Fig. 4).

\section{Discussion}

The current study was aimed to explore the association between miR-21 and let-7g expression patterns and the diagnostic outcome of CRC. Recent studies have proven the relationship between miRNA dysfunction/dysregulation and human diseases, including cancer. Despite that major number of miRNAs are intracellular, a large number of theses regulatory have been found to be present in different body 
fluids as circulating miRNAs. Their structure are chemically stable in cell culture supernatants and body fluids such as saliva, urine, blood and milk [29]. Results from new studies show that circulating miRNAs could act as potential diagnostic and prognostic markers in different types of cancers and other diseases. Additionally, early and timely diagnosis of cancer is one of the main objectives of cancer research projects worldwide. Thus, there has been a need for non-invasive biomarkers that would improve the early recognition of various types of cancer including CRC [30,31]. Furthermore, the expression pattern of miRNAs in the circulation could be related to the risk of disease development in CRC [32].

Wang and colleagues previously examined the expression profile of circulating miRNA in CRC patients [16]. They evidenced that some of circulating miRNAs, including miR-203 e.g. miR-31, miR-21, miR-92a, let-7g and miR-181b show dysregulated expression patterns CRC samples compared to healthy cases. Besides, the expression profile of these miRNAs was suggested to possess great value to identify CRC patients with high specificity $[16,33]$.

Additionally, our study further confirmed the diagnostic value of circulating miRNAs for screening of people with CRC risk. The results gathered using qRT-PCR showed that despite no significant change in stool samples, the expression levels of miR-21 and let-7g were remarkably dysregulated in serum samples of CRC patients. The results showed that miR-21 is highly expressed and let-7g is significantly down-regulated in in CRC serum samples, which was in consistence with previous findings[34]

Also, recent studies have shown that increased expression of miR-21 in patients with lung cancer can be used as a serum indicator to diagnose lung cancer. While the cancer studied in this study is completely different from the present study, both studies emphasize the role of this gene as a biomarker and introduce it as a useful tool for cancer diagnosis [35].

In a study conducted by Musilova $\mathrm{K}$ et al. On ovarian cancer, as in our results, the expression of let-7 in this cancer is reduced compared to control. In this study, in contrast to our study, which showed an increased amount of this microRNA in cancer tissue, the expression of this gene in cancer cells showed a significant decrease, which indicates the role of tumor suppression in this cancer. This difference in the results between the two studies can have different causes, the most important of which is the difference in the type of malignancy studied in the two studies and also the different biological roles of microRNAs. Another major difference between the two studies is the type of cancer being analyzed. In our study, colon cancer was selected and evaluated as a target, while in the study of Musilova K, et al., Ovarian cancer was evaluated as the target [36].

\section{Conclusion}

Our findings indicate that the expression patterns of miR-21 and let-7g gene can be considered as a diagnostic/predictive target in CRC serum samples. While, this is not the case in stool samples. Moreover, further investigations at the protein level should be performed. 


\section{Declarations}

\section{Ethics approval and consent to participate}

This study was confirmed by Ethical Committee of Tabriz University of Medical Sciences (Permit number: IR.TBZMED.REC.1397.441) and written informed consent was obtained from all the patients.

\section{Consent for publication}

Written informed consent was obtained from all patients.

\section{Availability of data and material}

All data in this article have been sent to the journal.

\section{Conflict of interests}

The authors declare that they have no competing interests.

\section{Funding}

The Ministry of Health and Medical Education, Deputy of Research and Technology, Iran, Tehran, has supported the study with funding a research grant with Grant number: 700/98, 2015.03.14 [1394/12/24]). Besides, the Tuberculosis and Lung Disease Research Center, Tabriz University of Medical Sciences has also supported the current study (ID: 60156).

\section{Authors' contributions}

(I) Conception and design: Dara Rahmanpour and Shahryar Hashemzadeh

(II) Administrative support: Roya Dolatkhah

(III) Provision of study materials or patients: Soheil Navidizadeh

(IV) Collection and assembly of data: Peyman Virani

(V) Data analysis and interpretation: Nastran Hasanzadeh and Habib Zarredar

(VI) Manuscript writing: Dara Rahmanpour and Shahryar Hashemzadeh

(VII) Final approval of manuscript: All Author

\section{Acknowledgements}

All authors contributed to this study are thankful from patients and their families for their cooperation. 


\section{References}

1. Fang, Q., et al., Expression and clinical significance of miR-210 in the serum of patients with colorectal cancer. Journal of Shandong University (Health Sciences), 2015. 53: p. 77-81.

2. Jemal, A., et al., Cancer statistics. Ca Cancer J Clin, 2009. 59(4).

3. Luo, X., et al., MicroRNA signatures: novel biomarker for colorectal cancer? Cancer Epidemiology and Prevention Biomarkers, 2011. 20(7): p. 1272-1286.

4. Siegel, R., D. Naishadham, and A. Jemal, Cancer statistics for hispanics/latinos, 2012. CA: a cancer journal for clinicians, 2012. 62(5): p. 283-298.

5. Hamada, T., R. Nishihara, and S. Ogino, Post-colonoscopy colorectal cancer: the key role of molecular pathological epidemiology. Translational gastroenterology and hepatology, 2017. 2.

6. Anvarnia, A., et al., Dysregulated microRNAs in colorectal carcinogenesis: new insight to cell survival and apoptosis regulation. Journal of cellular physiology, 2019. 234(12): p. 21683-21693.

7. Asadi, M., et al., Transcript level of MicroRNA processing elements in gastric cancer. Journal of gastrointestinal cancer, 2019. 50(4): p. 855-859.

8. Fang, Z., et al., Plasma levels of microRNA-24, microRNA-320a, and microRNA-423-5p are potential biomarkers for colorectal carcinoma. Journal of experimental \& clinical cancer research, 2015. 34(1): p. 110.

9. Mosher, C.E., et al., Associations between lifestyle factors and quality of life among older longterm breast, prostate, and colorectal cancer survivors. Cancer: Interdisciplinary International Journal of the American Cancer Society, 2009. 115(17): p. 4001-4009.

10. Sun, Y., et al., Examining plasma microRNA markers for colorectal cancer at different stages. Oncotarget, 2016. 7(10): p. 11434.

11. Wang, S., et al., A plasma microRNA panel for early detection of colorectal cancer. International journal of cancer, 2015. 136(1): p. 152-161.

12. Reinhart, B.J., et al., The 21-nucleotide let-7 RNA regulates developmental timing in Caenorhabditis elegans. nature, 2000. 403(6772): p. 901-906.

13. Mizuno, R., K. Kawada, and Y. Sakai, The molecular basis and therapeutic potential of Let-7 microRNAs against colorectal cancer. Canadian Journal of Gastroenterology and Hepatology, 2018. 2018.

14. Hormones, E. and B.C.C. Group, Endogenous sex hormones and breast cancer in postmenopausal women: reanalysis of nine prospective studies. Journal of the National Cancer Institute, 2002. 94(8): p. 
606-616.

15. Luo, X., et al., Identification and evaluation of plasma microRNAs for early detection of colorectal cancer. PloS one, 2013. 8(5): p. e62880.

16. Wang, B., et al., miR-29b suppresses tumor growth and metastasis in colorectal cancer via downregulating Tiam1 expression and inhibiting epithelial-mesenchymal transition. Cell death \& disease, 2014. 5(7): p. e1335-e1335.

17. Asangani, I.A., et al., MicroRNA-21 (miR-21) post-transcriptionally downregulates tumor suppressor Pdcd 4 and stimulates invasion, intravasation and metastasis in colorectal cancer. Oncogene, 2008. 27(15): p. 2128-2136.

18. Meng, F., et al., MicroRNA-21 regulates expression of the PTEN tumor suppressor gene in human hepatocellular cancer. Gastroenterology, 2007. 133(2): p. 647-658.

19. Zhu, S., et al., MicroRNA-21 targets the tumor suppressor gene tropomyosin 1 (TPM1). Journal of Biological Chemistry, 2007. 282(19): p. 14328-14336.

20. Kulda, V., et al., Relevance of miR-21 and miR-143 expression in tissue samples of colorectal carcinoma and its liver metastases. Cancer genetics and cytogenetics, 2010. 200(2): p. 154-160.

21. Nielsen, B.S., et al., High levels of microRNA-21 in the stroma of colorectal cancers predict short disease-free survival in stage II colon cancer patients. Clinical \& experimental metastasis, 2011. 28(1): p. 27-38.

22. Schetter, A.J., et al., MicroRNA expression profiles associated with prognosis and therapeutic outcome in colon adenocarcinoma. Jama, 2008. 299(4): p. 425-436.

23. Shibuya, $\mathrm{H}_{\text {., }}$ et al., Clinicopathological and prognostic value of microRNA-21 and microRNA-155 in colorectal cancer. Oncology, 2010. 79(3-4): p. 313-320.

24. Asaga, S., et al., Direct serum assay for microRNA-21 concentrations in early and advanced breast cancer. Clinical chemistry, 2011. 57(1): p. 84-91.

25. Kanaan, Z., et al., Plasma miR-21: a potential diagnostic marker of colorectal cancer. Annals of surgery, 2012. 256(3): p. 544-551.

26. Zarredar, H., et al., Terminalia Catappa Extract (TCE) Reduces Proliferation of Lung and Breast Cancer Cell by Modulating miR-21 and miR-34a Expressions. Asian Pacific Journal of Cancer Prevention, 2021. 22(4): p. 1157-1163.

27. Sotiriou, C., et al., Gene expression profiling in breast cancer: understanding the molecular basis of histologic grade to improve prognosis. Journal of the National Cancer Institute, 2006. 98(4): p. 262-272. 
28. O'Malley, M.S., et al., The association of race/ethnicity, socioeconomic status, and physician recommendation for mammography: who gets the message about breast cancer screening? American Journal of Public Health, 2001. 91(1): p. 49.

29. Mitchell, P.S., et al., Circulating microRNAs as stable blood-based markers for cancer detection. Proceedings of the National Academy of Sciences, 2008. 105(30): p. 10513-10518.

30. Chen, X., et al., Characterization of microRNAs in serum: a novel class of biomarkers for diagnosis of cancer and other diseases. Cell research, 2008. 18(10): p. 997-1006.

31. Gilad, S., et al., Serum microRNAs are promising novel biomarkers. PloS one, 2008. 3(9): p. e3148.

32. Arefi, M., et al., Expression Analysis of Circulating miR-21 in Iranian Patients with Hereditary Colorectal Cancer. Medical Laboratory Journal, 2021. 15(2): p. 1-4.

33. Moridikia, A., et al., MicroRNAs: Potential candidates for diagnosis and treatment of colorectal cancer. Journal of cellular physiology, 2018. 233(2): p. 901-913.

34. Jin, X.-H., S. Lu, and A.-F. Wang, Expression and clinical significance of miR-4516 and miR-21-5p in serum of patients with colorectal cancer. BMC cancer, 2020. 20(1): p. 1-7.

35. Zhong, S., et al., Relationship between the GSTM1 genetic polymorphism and susceptibility to bladder, breast and colon cancer. Carcinogenesis, 1993. 14(9): p. 1821-1824.

36. Musilová, K. and M. Mráz, MicroRNAs in B-cell lymphomas: how a complex biology gets more complex. Leukemia, 2015. 29(5): p. 1004-1017.

\section{Figures}

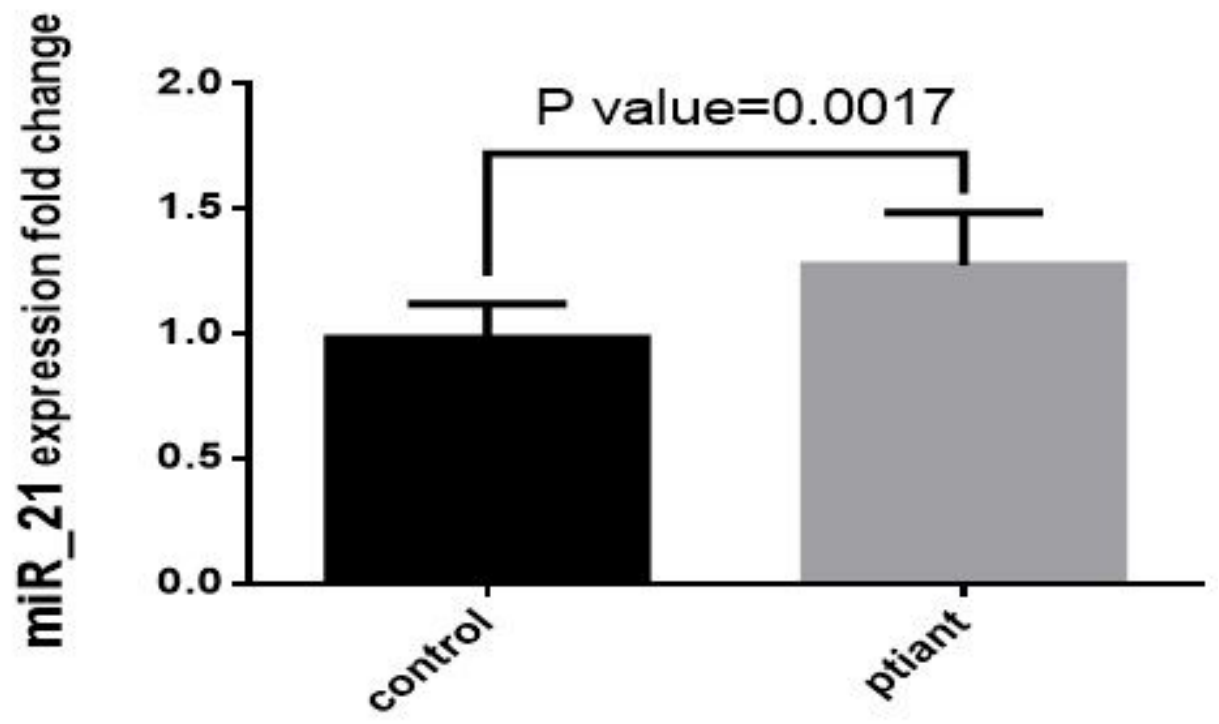


The alteration of expression level of mir-21 in serum of colon cancer patients compared with healthy group.

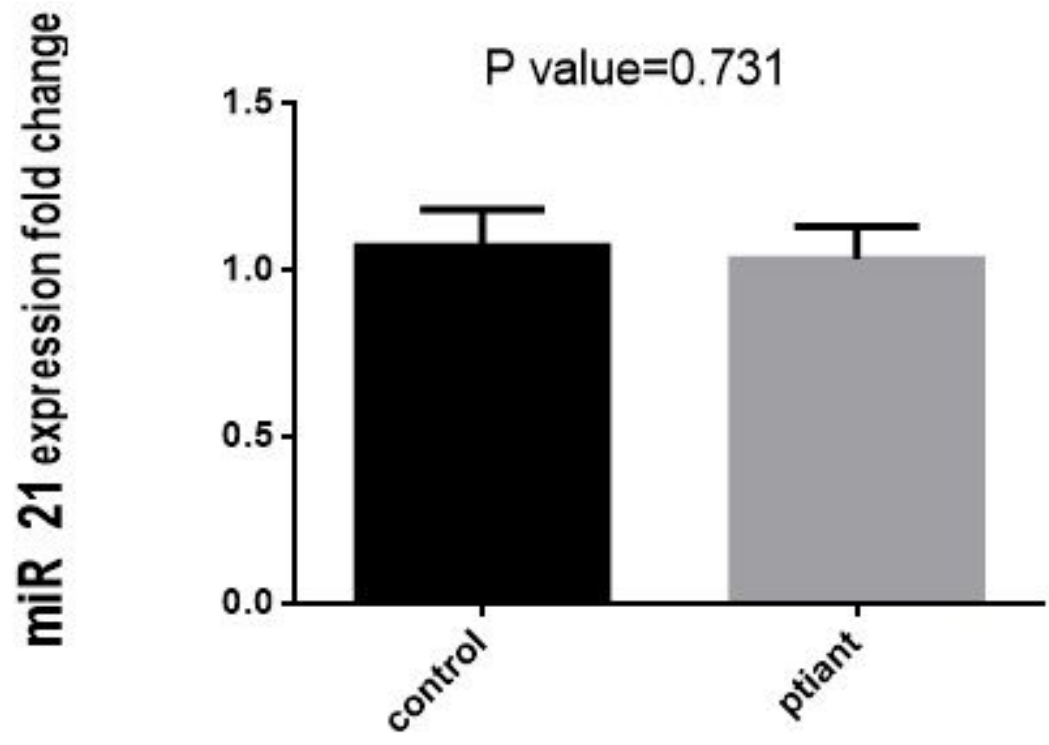

Figure 2

The alteration of expression level of mir-21 in stool samples of patients compared with healthy group.

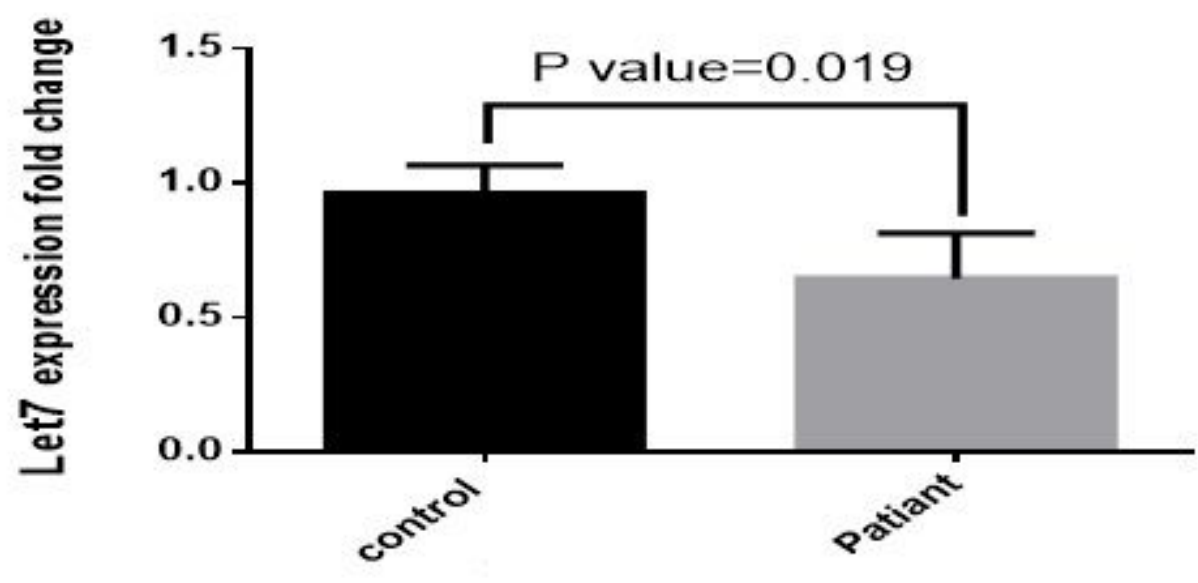

Figure 3

The alteration of expression level of let-7g in serum samples of patients compared with healthy group. 


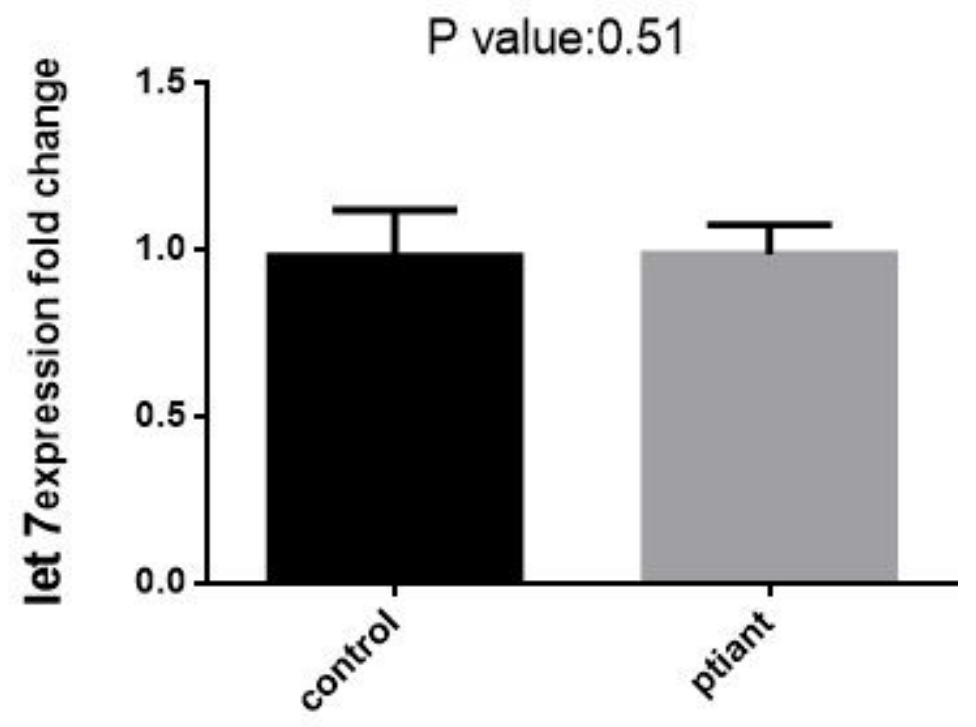

Figure 4

The alteration of expression level of let-7g in stool samples of patients compared with healthy group. 